\title{
Hawking radiation of Dirac particles via tunneling from Kerr black hole
}

\author{
Ran Li:* Ji-Rong Ren $\dagger$ and Shao-Wen Wei \\ Institute of Theoretical Physics, Lanzhou University, Lanzhou, 730000, Gansu, China
}

\begin{abstract}
We investigated Dirac Particles' Hawking radiation from event horizon of Kerr black hole in terms of the tunneling formalism. Applying WKB approximation to the general covariant Dirac equation in Kerr spacetime background, we obtain the tunneling probability for fermions and Hawking temperature of Kerr black hole. The result obtained by taking the fermion tunneling into account is consistent with the previous literatures.

PACS numbers: 04.70.Dy, 03.65.Sq
\end{abstract}

Keywords: tunneling, Hawking radiation, Kerr black hole

${ }^{*}$ Electronic mail: liran05@lzu.cn

${ }^{\dagger}$ Corresponding author. Electronic mail:renjr@lzu.edu.cn 
Hawking[1] discovered the thermal radiation of a collapsing black hole using the techniques of quantum field theory in curved spacetime. Since the Hawking radiation relates the theory of general relativity with quantum field theory and statistical thermodynamics, it is generally believed that a deeper understanding of Hawking radiation may shed some lights on seeking the underlying quantum gravity. Since then, several derivations of Hawking radiation have been proposed. The original method presented by Hawking is direct but complicated to be generalized to other spacetime backgrounds. In recent years, a semiclassical derivation of Hawking radiation as a tunneling process [2] has been developed and has already attracted a lot of attention. In this method, the imaginary part of the action is calculated using the null geodesic equation. Zhang and Zhao extended this method to Reissner-Nordström black hole[3] and Kerr-Newman black hole[4]. M. Angheben et al [5] also proposed a derivation of Hawking radiation by calculating the particles' classical action from the Hamilton-Jacobi equation, which is an extension of the complex path analysis of T. Padmanabhan et al [6]. All of these approaches to tunneling used the fact that the tunneling probability for the classically forbidden trajectory from inside to outside the horizon is given by

$$
\Gamma=\exp \left(-\frac{2}{\hbar} \operatorname{Im} I\right)
$$

where $I$ is the classical action of the trajectory. The crucial thing in tunneling formalism is to calculate the imaginary part of classical action. The difference between these two methods consists in how the classical action is calculated. For a detailed comparison of the Hamilton-Jacobi method and the Null-Geodesic method, one can see [7].

However, most of authors only considered the scalar particles' radiation. Very recently, a new calculation concerning fermions' radiation from the stationary spherical symmetric black hole was done by R. Kerner and R. B. Mann in [8]. This method have been generalized to BTZ black hole by us in [9] and dynamical black hole in [10]. In this letter, we will generalize the tunneling method presented in [8] to calculate the Dirac particles' Hawking radiation from Kerr black hole. Starting with the general covariant Dirac equation in curved background, we calculate the tunneling probability and Hawking temperature by using WKB approximation. The result obtained by taking the fermion tunneling into account is consistent with the previous literatures. 
The Kerr black hole solution in Boyer-Linquist coordinates is given by

$$
\begin{aligned}
d s^{2}= & -\frac{\Delta-a^{2} \sin ^{2} \theta}{\Sigma} d t^{2}-2 a \sin ^{2} \theta \cdot \frac{r^{2}+a^{2}-\Delta}{\Sigma} d t d \phi \\
& +\frac{\left(r^{2}+a^{2}\right)^{2}-\Delta a^{2} \sin ^{2} \theta}{\Sigma} \cdot \sin ^{2} \theta d \phi^{2}+\frac{\Sigma}{\Delta} d r^{2}+\Sigma d \theta^{2}
\end{aligned}
$$

where

$$
\begin{aligned}
\Sigma & =r^{2}+a^{2} \cos ^{2} \theta, \\
\Delta & =r^{2}-2 M r+a^{2}=\left(r-r_{+}\right)\left(r-r_{-}\right), \\
r_{ \pm} & =M \pm \sqrt{M^{2}-a^{2}} .
\end{aligned}
$$

We have assumed the non-extremal condition $M>a$, so that $r_{+}$and $r_{-}$correspond to the outer event horizon and the inner event horizon respectively. The determinant of the metric is

$$
\sqrt{-g}=\Sigma \sin \theta
$$

and the inverse of the metric of $(t, \phi)$ parts is

$$
\begin{aligned}
g^{t t} & =-\frac{\left(r^{2}+a^{2}\right)^{2}-\Delta a^{2} \sin ^{2} \theta}{\Sigma \Delta}, \\
g^{\phi \phi} & =\frac{\Delta-a^{2} \sin ^{2} \theta}{\Sigma \Delta \sin ^{2} \theta}, \\
g^{t \phi} & =-\frac{a\left(r^{2}+a^{2}-\Delta\right)}{\Sigma \Delta} .
\end{aligned}
$$

Now we calculate the Dirac particles' Hawking radiation from the outer event horizon of Kerr black hole via tunneling formulism. For simplicity, we only consider the massless spinor field $\Psi$ obeys the general covariant Dirac equation

$$
-i \hbar \gamma^{a} e_{a}^{\mu} \nabla_{\mu} \Psi=0
$$

where $\nabla_{\mu}$ is the spinor covariant derivative defined by $\nabla_{\mu}=\partial_{\mu}+\frac{1}{4} \omega_{\mu}^{a b} \gamma_{[a} \gamma_{b]}$, and $\omega_{\mu}^{a b}$ is the spin connection, which can be given in terms of the tetrad $e_{a}^{\mu}$. The $\gamma$ matrices are selected 
as

$$
\begin{aligned}
& \gamma^{0}=\left(\begin{array}{cc}
i & 0 \\
0 & -i
\end{array}\right), \\
& \gamma^{1}=\left(\begin{array}{cc}
0 & \sigma^{3} \\
\sigma^{3} & 0
\end{array}\right), \\
& \gamma^{2}=\left(\begin{array}{ll}
0 & \sigma^{1} \\
\sigma^{1} & 0
\end{array}\right), \\
& \gamma^{3}=\left(\begin{array}{cc}
0 & \sigma^{2} \\
\sigma^{2} & 0
\end{array}\right),
\end{aligned}
$$

where the matrices $\sigma^{k}(k=1,2,3)$ are the Pauli matrices. According to the line element (2), the tetrad fields $e_{a}^{\mu}$ can be selected to be

$$
\begin{aligned}
e_{0}^{\mu} & =\left(\sqrt{-g^{t t}}, 0,0, \frac{-g^{t \phi}}{\sqrt{-g^{t t}}}\right), \\
e_{1}^{\mu} & =\left(0, \sqrt{\frac{\Delta}{\Sigma}}, 0,0\right) \\
e_{2}^{\mu} & =\left(0,0, \frac{1}{\sqrt{\Sigma}}, 0\right) \\
e_{3}^{\mu} & =\left(0,0,0, \frac{1}{\sqrt{g_{\phi \phi}}}\right) .
\end{aligned}
$$

We employ the ansatz for the spin-up spinor field $\Psi$ as following [8, 10]

$$
\Psi=\left(\begin{array}{c}
A(t, r, \theta, \phi) \\
0 \\
B(t, r, \theta, \phi) \\
0
\end{array}\right) \exp \left[\frac{i}{\hbar} I(t, r, \theta, \phi)\right] .
$$

Note that we will only analysis the spin-up case since the spin-down case is just analogous. In order to apply WKB approximation, we can insert the ansatz for spinor field $\Psi$ into the general covariant Dirac equation. Dividing by the exponential term and neglecting the terms with $\hbar$, one can arrive at the following four equations

$$
\left\{\begin{array}{l}
i A\left(\sqrt{-g^{t t}} \partial_{t} I-\frac{g^{t \phi}}{\sqrt{-g^{t t}}} \partial_{\phi} I\right)+B \sqrt{\frac{\Delta}{\Sigma}} \partial_{r} I=0 \\
\left(\frac{1}{\sqrt{\Sigma}} \partial_{\theta} I+i \frac{1}{\sqrt{g_{\phi \phi}}} \partial_{\phi} I\right) B=0 \\
A \sqrt{\frac{\Delta}{\Sigma}} \partial_{r} I-i B\left(\sqrt{-g^{t t}} \partial_{t} I-\frac{g^{t \phi}}{\sqrt{-g^{t t}}} \partial_{\phi} I\right)=0 \\
\left(\frac{1}{\sqrt{\Sigma}} \partial_{\theta} I+i \frac{1}{\sqrt{g_{\phi \phi}}} \partial_{\phi} I\right) A=0 .
\end{array}\right.
$$


Note that although $A$ and $B$ are not constant, their derivatives and the components $\omega_{\mu}$ are all of the factor $\hbar$, so can be neglected to the lowest order in WKB approximation. Because we only consider the Dirac field outside the event horizon, the condition $\Delta \geq 0$ is always satisfied in the above equations. The second and fourth equations indicate that

$$
\frac{1}{\sqrt{\Sigma}} \partial_{\theta} I+i \frac{1}{\sqrt{g_{\phi \phi}}} \partial_{\phi} I=0
$$

From the first and third equations one can see that these two equations have a non-trivial solution for $A$ and $B$ if and only if the determinant of the coefficient matrix vanishes. Then we can get

$$
\left(\sqrt{-g^{t t}} \partial_{t} I-\frac{g^{t \phi}}{\sqrt{-g^{t t}}} \partial_{\phi} I\right)^{2}-\frac{\Delta}{\Sigma}\left(\partial_{r} I\right)^{2}=0 .
$$

Because there are two killing vectors $(\partial / \partial t)^{\mu}$ and $(\partial / \partial \phi)^{\mu}$ in Kerr-Newman spacetime, we can separate the variables for $I(t, r, \theta, \phi)$ as following

$$
I=-\omega t+j \phi+R(r, \theta)+K,
$$

where $\omega$ and $j$ are Dirac particle's energy and angular momentum respectively and $K$ is a complex constant. Inserting it to equation (7), one can arrive

$$
\left(\sqrt{-g^{t t}} \omega+\frac{g^{t \phi}}{\sqrt{-g^{t t}}} j\right)^{2}-\frac{\Delta}{\Sigma}\left(\partial_{r} R\right)^{2}=0 .
$$

Equation (6) indicates that $R(r, \theta)$ is a complex function. Now solving the equation (9) for definite $\theta_{0}$ yields [5, 7]

$$
\begin{aligned}
R_{ \pm}\left(r, \theta_{0}\right) & = \pm \int d r \sqrt{\frac{\Sigma\left(\theta_{0}\right)}{\Delta}}\left(\sqrt{-g^{t t}\left(\theta_{0}\right)} \omega+\frac{g^{t \phi}\left(\theta_{0}\right)}{\sqrt{-g^{t t}\left(\theta_{0}\right)}} j\right) \\
& = \pm \int \frac{d r}{\Delta}\left(\omega \sqrt{\left(r^{2}+a^{2}\right)^{2}-\Delta a^{2} \sin ^{2} \theta_{0}}-j \frac{a\left(r^{2}+a^{2}-\Delta\right)}{\sqrt{\left(r^{2}+a^{2}\right)^{2}-\Delta a^{2} \sin ^{2} \theta_{0}}}\right)
\end{aligned}
$$

The imaginary part of $R_{+}$can be calculated using the above equation. Integrating the pole at the horizon leads to the result (see [7, 12] for a detailed similar process)

$$
\operatorname{Im} R_{ \pm}= \pm \frac{\pi\left(r^{2}+a^{2}\right)}{r_{+}-r_{-}}\left(\omega-j \Omega_{H}\right)
$$

where $\Omega_{H}=\frac{a}{r_{+}^{2}+a^{2}}$ is the angular velocity of event horizon. One can see that this result is independent of $\theta$. 
As discussed in the Hamilton-Jacobi method[11, 12], one solution corresponds Dirac particles moving away from the outer event horizon and the other solution corresponds the particles moving toward the outer event horizon. The probabilities of crossing the outer horizon each way are respectively given by

$$
\begin{aligned}
P_{\text {out }} & =\exp \left[-\frac{2}{\hbar} \operatorname{Im} I\right]=\exp \left[-\frac{2}{\hbar}\left(\operatorname{Im} R_{+}+\operatorname{Im} K\right)\right] \\
P_{\text {in }} & =\exp \left[-\frac{2}{\hbar} \operatorname{Im} I\right]=\exp \left[-\frac{2}{\hbar}\left(\operatorname{Im} R_{-}+\operatorname{Im} K\right)\right] .
\end{aligned}
$$

To ensure that the probability is normalized, we should note that the probability of any incoming wave crossing the outer horizon is unity[12]. So we get $\operatorname{Im} K=-\operatorname{Im} R_{-}$. Since $\operatorname{Im} R_{+}=-\operatorname{Im} R_{-}$this implies that the probability of a Dirac particle tunneling from inside to outside the evnet horizon is given by

$$
\begin{aligned}
\Gamma & =\exp \left[-\frac{4}{\hbar} \operatorname{Im} R_{+}\right] \\
& =\exp \left[\frac{4 \pi\left(r_{+}^{2}+a^{2}\right)}{\left(r_{+}-r_{-}\right)}\left(\omega-j \Omega_{H}\right)\right],
\end{aligned}
$$

where in the last step we set $\hbar$ to unity. It should be noted that the higher terms about $\omega$ and $j$ are neglected in our derivation and the expression (12) for tunneling probability implies the pure thermal radiation.

From the tunneling probability, the fermionic spectrum of Hawking radiation of Dirac particles from Kerr black hole can be deduced following the standard arguments [13, 14]

$$
N(\omega, j)=\frac{1}{e^{2 \pi\left(\omega-j \Omega_{H}\right) / \kappa}+1}
$$

where $\kappa=\frac{\left(r_{+}-r_{-}\right)}{2\left(r_{+}^{2}+a^{2}\right)}$ is the surface gravity of event horizon. From the tunneling probability and radiant spectrum, Hawking temperature of Kerr black hole can be determined as

$$
T=\frac{\kappa}{2 \pi}=\frac{\sqrt{M^{2}-a^{2}}}{4 \pi M\left(M+\sqrt{M^{2}-a^{2}}\right)} .
$$

In summary, we have calculated the Dirac particles' Hawking radiation from Kerr black hole using the tunneling formalism. Starting with Dirac equation, we obtained the radiation spectrum and Hawking temperature of Kerr black hole by using the WKB approximation. The results coincide with the previous literatures [4, 5, 7, 15, 16]. 


\section{ACKNOWLEDGEMENT}

After completing this paper, we noticed that some related works were done by other

authors. In 17], charged Fermions tunnelling from Kerr-Newman black holes were investigated. In [18], the authors considered Fermions tunnelling from the more general and complicated spacetime background. This work was supported by the National Natural Science Foundation of China and Cuiying Project of Lanzhou University.

[1] S.W.Hawking, Commun.Math.Phys.43,199(1975).

[2] M.K.Parikh and F.Wilczek, Phys.Rev.Lett.85,5042(2000).

[3] J.Zhang and Z.Zhao, JHEP 10,055(2005).

[4] J.Zhang and Z.Zhao, Phys.Lett.B 618,14(2005); J.Zhang and Z.Zhao, Phys.Lett.B $638,110(2006)$.

[5] M.Angheben, M.Nadalini, L.Vanzo and S.zerbini, JHEP 05, 014(2005).

[6] K.Srinivasan and T.Padmanabhan, Phys.Rev.D 60, 24007(1999); S.Shankaranarayanan, T.Padmanabhan and K.Srinivasan, Class.Quant.Grav. 19, 2671(2002).

[7] R.Kerner and R.B.Mann, Phys.Rev.D 73, 104010(2006).

[8] R.Kerner and R.B.Mann, Class.Quant.Grav. 25, 095014(2008).

[9] R. Li, J. R. Ren, Phys. Lett. B, 661, 370(2008).

[10] R. Di Criscienzo and L. Vanzo, arXiv: 0803.0435.

[11] E.T.Akhmedov, V.Akhmedov and D.Singleton, Phys.Lett.B 642, 124(2006).

[12] P.Mitra, Phys.Lett.B 648, 240(2007).

[13] T.Damour and R.Ruffini, Phys.Rew.D 14, 332(1976).

[14] S.Sannan, Gen.Rel.Grav. 20, 139(1988).

[15] Q.Jiang, S.Wu and X.Cai, Phys.Rev.D 73, 064003(2006).

[16] S.P.Kim, JHEP, 11, 048(2007).

[17] R. Kerner and R.B. Mann, arXiv:0803.2246.

[18] D. Chen, Q. Jiang, S. Yang and X. Zu, arXiv:0803.3248; D. Chen, Q. Jiang and X. Zu, arXiv:0804.0131. 